\title{
A Load Identification Technology for Wireless Smart Home Systems
}

\author{
Zhenchao Xie ${ }^{1, *}$, Daohong Lin ${ }^{1}$, Yufeng Chen ${ }^{2}$ and Xinshu Wan ${ }^{1}$ \\ ${ }^{1}$ Electric Power Research Institute of Hainan Power Grid Co., Ltd., Haikou 570125, Hainan Province, China \\ ${ }^{2}$ Nanjing Sifang E-power Automation Co., Ltd., Nanjing 211111, China \\ ${ }^{*}$ Corresponding author
}

\begin{abstract}
Aiming at the applications in wireless smart homes, a wireless charging system based on LCL topology is designed. Moreover, the constant-current (CC) output of the system is realized according to the charging characteristics of lithium batteries. On this basis, a charging strategy is proposed, which is able to identify load characteristics and take corresponding actions after the access of loads in smart homes to the system, and can guarantee the safety of the loads in power supply process. Finally, an experimental prototype of the system was established to wirelessly charge the lithium battery with a nominal voltage of $7.4 \mathrm{~V}$ and verify the validity of the proposed charging strategy for protecting the system when illegal loads were connected in the system.
\end{abstract}

Keywords—wireless power transfer; load identification; lithium battery; charging strategy

\section{INTRODUCTION}

Research on the wireless power transfer (WPT) technology has never stopped ever since the proposal of the technology. Compared with traditional wire power transfer approaches, WPT has many advantages, such as being more flexible, safe, and reliable. It avoids potential safety hazards such as electric sparks of wired connection [1-4]. Characterized by these advantages, the WPT technology has been widely used in smart homes. At present, many smart homes are powered by lithium batteries, the whole charging process of which has two phases: $\mathrm{CC}$ charging and constant-voltage $(\mathrm{CV})$ charging processes [5].

In general, to realize the control over the $\mathrm{CC}$ and $\mathrm{CV}$ charging of lithium batteries, a specific direct current (DC) $\mathrm{CV} / \mathrm{CC}$ conversion circuit needs to be designed [6-7]. For example, $\mathrm{CV}$ power supply is provided for handheld devices by adding DC converters in the secondary side of the wireless charging system in previous research [6]. Existing studies also found that resonant WPT systems have inherent CC characteristics under specific topological structures and frequencies. If the topological structures of WPT systems can be used to realize the $\mathrm{CC}$ charging of lithium batteries, it is of great significance for reducing the complexity of the back-end circuits of the systems.

Meanwhile, to guarantee the operation safety of wireless smart home systems, corresponding protections need to be designed for the systems. As the transmission characteristics of WPT systems are related to the system loads (batteries), the random access of batteries of different voltage classes is disadvantage for the safe operation of wireless systems with given parameters. If we can detect the properties and sizes of loads after the loads are connected to the system and then control the system on this basis, the safety of the system can be improved. Aiming at the problem, an effective identification method for loads was put forward in previous study [8]. The method allows one to detect the sizes and properties of loads connected to the systems by means of the energy injection mode and the free harmonic vibration mode.

Based on wireless smart home systems, the research designs a wireless charging system. The LCL compensation network is always in the primary side of the system. By reasonably designing the compensation network in the primary side, the LCL-LCL wireless system is formed, which is used for controlling the $\mathrm{CC}$ charging of lithium batteries. Then, a charging strategy of the system is proposed based on the reflection and impedance characteristics of the system. The charging strategy enables the system to identify illegal loads and improves the safety of the system. Finally, an experimental prototype of the system was built to carry out wireless charging of the lithium battery with a nominal voltage of $7.4 \mathrm{~V}$ and verify the validity of the proposed charging strategy after the access of illegal loads to the system.

\section{Design OF THE WIRELESS CHARgING SySteM}

When factors including the internal resistance are ignored, the schematic diagram of the ideal LCL circuit is illustrated in Figure I, where $U_{\text {in }}$ represents the input power, $L_{X}$ and $L_{\mathrm{p}}$ denote inductances, $C_{P}$ refers to compensating capacitor, and $Z$ represents equivalent load. Let the parameters $L_{X}=L_{\mathrm{p}}$ and $\omega_{0}=\operatorname{sqrt}\left(1 / L_{p} C_{P}\right)$, the current $i_{L p}$ flowing through the load $Z$ is $U_{\text {in }} /\left(j \omega_{0} L_{P}\right)$ when the system works at the resonant angular frequency $\omega_{0}$.

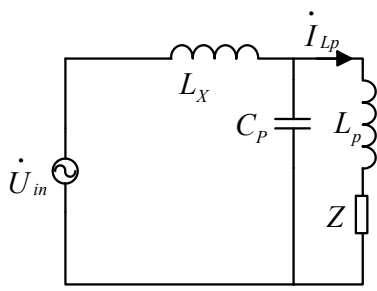

FIGURE I.

SCHEMATIC DIAGRAM OF THE CIRCUIT BASED ON LCL TOPOLOGY

Under the condition, the current $i_{L p}$ of the load is irrelevant to $Z$ but only related to the power source $U_{\text {in }}$, inductance $L_{P}$, 
and the resonant angular frequency $\omega_{0}$. That is, the branch current in the LCL circuit where the inductance is has CC characteristics. Suppose that the CC characteristics of LCL topology are combined with WPT systems, LCL topology is taken as the topological structure of the wireless energy transmit side, and the inductance $L_{\mathrm{p}}$ is assumed as the equivalent inductance of the transmit coil. Under the condition, it is equivalent to add a voltage source with the voltage of $j \omega M I_{\mathrm{Lp}}$ in the branch of the receiving coil (inductance) $L_{\mathrm{s}}$ in the receiving side according to the $M$ model. The value of the voltage source is merely influenced by the power source in the transmit side but not impacted by the circuit parameters in the receiving side.

To endow the loads in the receiving side with the CC output characteristics, it is necessary to further design the topological structure in the receiving side. According to the connection mode of coils and capacitors, the authors design the LCL topology for the receiving side, as illustrated in Figure II. In the figure, $\mathbf{U}_{\text {in }}$ represents a high-frequency alternating current (AC) power source, $L_{X}$ and $L_{Y}$ are the inductances of the compensating inductor, $C_{\mathrm{p}}$ and $C_{\mathrm{S}}$ denote the capacitances of the compensating capacitor, $L_{\mathrm{P}}$ and $L_{\mathrm{S}}$ represent the inductances of the transmit and the receiving coils, $M$ refers to the mutual inductance between the transmit and receiving coils. A microprocessor MCU1 is placed in the primary side of the system to control the value of the input voltage $\mathbf{U}_{\text {in }}$ by detecting the effective value of the voltages of the compensating capacitor in the primary side. A switch $\mathrm{S}_{4}$ is set in the secondary side to protect the system and controlled by the microprocessor MCU2.

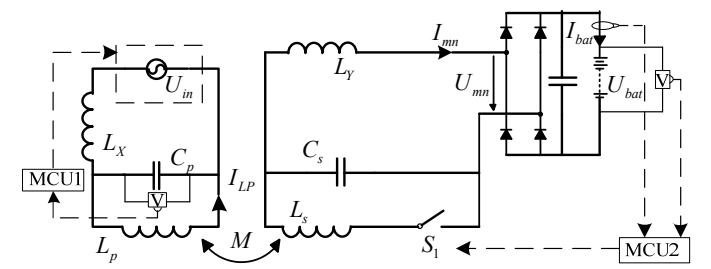

FIGURE II.

EQUIVALENT CIRCUIT DIAGRAM OF THE WIRELESS CHARGING SYSTEM

Let the inductances be $L_{\mathrm{X}}=L_{\mathrm{Y}}=L_{\mathrm{P}}=L_{\mathrm{S}}=L$, the capacitances be $C_{\mathrm{p}}=C_{\mathrm{S}}=C$, and the working angular frequency be $\omega=\operatorname{sqr}(1 / \mathrm{LC})$ in the system. While analyzing the output characteristics of the system in the section, it is assumed that the system works normally and the switch $\mathrm{S}_{4}$ is closed. In this way, a connection mode based on LCL-LCL topology is formed in the primary and secondary sides of the system. Then, the current $I_{L p}$ flowing through the transmit coil is

$$
\mathbf{I}_{\mathbf{L p}}=\mathbf{U}_{\mathrm{in}} / j \omega L
$$

Obviously, the value of $\mathbf{I}_{\mathbf{L p}}$ is merely related to the parameters in the primary side. According to the theory of mutual inductance, it is equivalent to introduce a constant controlled voltage source $j \omega M \mathbf{I}_{\mathbf{L p}}$ in the primary side of the system. In accordance with the calculation, it can be found that the current $\mathbf{I}_{\mathbf{m n}}$ flowing before the rectifier is

$$
\mathbf{I}_{\mathrm{mn}}=M \mathbf{U}_{\mathrm{in}} /\left(j \omega L^{2}\right)
$$

It can be seen that the current before the rectifier is not related to the size and properties of the loads. The following current relation before and after the rectifier can be obtained:

$$
I_{b a t}=2 \sqrt{2}\left|\mathbf{I}_{\mathbf{m n}}\right| / \pi
$$

Then, it can be learnt from Formulae (2) and (3) that based on the LCL-LCL topology, the current flowing through the battery is

$$
I_{b a t}=2 \sqrt{2} M\left|\mathbf{U}_{\text {in }}\right| /\left(\pi \omega L^{2}\right)
$$

Apparently, the charging current of the battery does not change after the parameters of the system are set. It means that the system achieves $\mathrm{CC}$ output in the connection mode based on LCL-LCL topology.

\section{Charging Strategy of The System}

While WPT technology is applied to charge batteries, WPT systems need to be designed with different parameters for different batteries. In other words, a designed WPT system can merely charge specific type of batteries. Other types of batteries cannot enter the system optionally. It is stipulated that in the wireless charging system in Figure II built according to the actual charging demand of batteries, the corresponding batteries are legal loads while other loads whose nominal voltages are different with the legal loads are illegal loads. As the nominal voltage of illegal loads has disparities with that of the legal loads and different batteries have dissimilar bearable charging currents, the access of illegal loads can probably damage the batteries. Therefore, it is necessary to analyze the protection of the loads after illegal loads enter a WPT system.

Suppose that for a lithium battery, its nominal voltage is

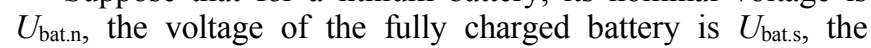
minimum voltage in under-voltage state is $U_{\text {bat,u }}$, and the charging current of the battery during CC charging is $I_{\text {bat,p. }}$. The external circuit of the lithium battery can be regarded as linking a DC power source in the two sides of the battery at any moment during the charging, and its voltage is the voltage $U_{\text {bat }}$ of the battery and the charging current is $I_{\text {bat. }}$ Therefore, the external circuit of the battery can be equivalent to a varying resistance $R_{\text {bat,eq }}=U_{\text {bat }} / I_{\text {bat }}$.

As shown in Figure II, the voltage of the power source in the primary side of the system is controlled by MCU1 which controls the switch of the power source between two working conditions: one is the normal working state, in which the output voltage of the inverter is $U_{\text {in }}$; and the other is standby state, in which the output voltage of the inverter is $U_{\mathrm{in}, \mathrm{s}}$, and $U_{\mathrm{in,s}}<<U_{\mathrm{in}}$. According to Formula (4), as the power source in the two states has different voltages, the charging current of the battery also changes in the system based on LCL-LCL topology. Therein, the charging current of the battery in normal working state is $I_{\text {bat,p. }}$. 


$$
I_{b a t, p}=2 \sqrt{2} M\left|\mathbf{U}_{\mathrm{in}}\right| /\left(\pi \omega L^{2}\right)
$$

In the standby state, the charging current is $I_{\text {bat,s. }}$.

$$
I_{b a t, s}=2 \sqrt{2} M \mid \mathbf{U}_{\mathrm{in}, \mathrm{s}} / /\left(\pi \omega L^{2}\right)
$$

On the basis of the above analysis, the identification of illegal loads and the protection measures for the system are shown as follows:

1) When no battery enters the system, the system is in the standby state and the output of the inverter is $\mathbf{U}_{\text {in,s. }}$ Under the condition, all of the switches in Figure I are turned off and the voltage in two sides of the compensating capacitor $\mathrm{C}_{\mathrm{p}}$ is $U_{\mathrm{in}, \mathrm{s}}$.

2) When there is battery connected to the system, the secondary side of the system detects the voltage $U_{\text {bat }}$ in two sides of the battery. If the voltage satisfies

$$
U_{\text {bat,u }}<U_{\text {bat }}<U_{\text {bat, }}
$$

then the secondary side believes that the battery entering the system is a legal load and the $\mathrm{S}_{1}$ is turned on under the control of MCU2. Under the condition, the system forms a LCL-LCL topology. The information of the primary side is independent to that of the secondary side, and the primary side identifies the load in the system by detecting the voltage in the two sides of the compensating capacitor $\mathrm{C}_{\mathrm{p}}$ in real time. Then the relation between the equivalent resistances before and after the known rectifier is

$$
R_{m n}=8 R_{b a t, e q} / \pi^{2}
$$

Where $R_{\mathrm{mn}}=\mathbf{U}_{\mathbf{m n}} / \mathbf{I}_{\mathbf{m n}}$. Based on this, it is obtained that the voltage in the two sides of the compensating capacitor $C_{p}$ and the load has the following relation in the LCL-LCL topology:

$$
\dot{\mathbf{U}}_{C p}=\dot{\mathbf{U}}_{i n}\left(1+\omega M^{2} C R_{m n} /\left(j L^{2}\right)\right)
$$

Then, the relation between the effective value of the voltage in the two sides of the compensating capacitor $\mathrm{C}_{\mathrm{p}}$ and the load is

$$
\left|U_{C p}\right|=\left|U_{i n}\right| \sqrt{\left(1+M^{4} R_{m n}^{2} /\left(\omega^{2} L^{6}\right)\right)}
$$

Formula (8) reveals that if the battery in the system is a legal load, then the equivalent resistance of the battery meets

$$
U_{b a t, u} / I_{b a t, s} \leq R_{b a t, e q} \leq U_{b a t, s} / I_{b a t, s}
$$

It is deduced that the equivalent resistance before rectification satisfies

$$
\frac{8 U_{b a t, u}}{\pi^{2} I_{b a t, s}} \leq R_{m n} \leq \frac{8 U_{b a t, s}}{\pi^{2} I_{b a t, s}}
$$

By substituting Formula (12) into (10), it is observed that if the primary side finds that $\left|\mathbf{U}_{\mathbf{C p}}\right|$ meets the following condition

$$
\left|\mathbf{U}_{\mathrm{in}, \mathrm{s}}\right| \sqrt{\left(1+\frac{64 M^{4} U_{b a t, u}^{2}}{\pi^{4} \omega^{2} L^{6} I_{b a t, s}^{2}}\right)} \leq\left|\mathbf{U}_{\mathbf{C p}}\right| \leq\left|\mathbf{U}_{\mathrm{in,s}}\right| \sqrt{\left(1+\frac{64 M^{4} U_{b a t, n}^{2}}{\pi^{4} \omega^{2} L^{6} I_{b a t, s}^{2}}\right)}
$$

then the primary side regards the battery as a legal load. Afterwards, the power source is switched to the normal working voltage $\mathbf{U}_{\text {in }}$ under the control of MCU1, turning the system to the normal working state.

3) If the battery entering the system is detected as an illegal load, the voltage in the two sides of the battery detected by the primary side of the system does not meet Formula (7), then the switch in the secondary side is turned off under the control of the MCU2. Under the condition, the capacitor voltage detected by the primary side of the system is still

$$
\left|\mathbf{U}_{\mathrm{Cp}}\right|=\left|\mathbf{U}_{\mathrm{in,s}}\right| .
$$

Under the control of MCU1, the output voltage of the inverter is still the standby voltage $U_{\mathrm{in}, \mathrm{s}}$, that is, the system is still in the standby state.

\section{EXPERIMENTAL VERIFICATION}

To verify the validity of the proposed charging strategy for batteries, the research took a lithium battery with a nominal voltage of $U_{\text {bat,n }}=7.4 \mathrm{~V}$ as the charging object. After being fully charged, the battery has an open-circuit voltage of $U_{\text {bat, } s}=8.4 \mathrm{~V}$. Let the charging current of the lithium battery in CC charging mode be $I_{\text {bat }}=1$ A. Based on the above charging parameters of the battery, the authors established the experimental prototype, and the parameters of the system are displayed in Table I. After building the system, the lithium battery became a legal load of the system. In the experimental prototype, the transmit and receiving coils of 12 turns were winded using Litz wires of 1.5 $\mathrm{mm}$ wide, and the spacing between them was $3 \mathrm{~cm}$. The bridge inverter circuit was taken for the inverter and the bridge driving chip UCC3895 was used to drive four IRFP460 MOS tubes. In the standby state, the input voltage $U_{\text {in,s }}$ of the system was $1 \mathrm{~V}$, while the input voltage $U_{\text {in }}$ in the normal working state was $36.5 \mathrm{~V}$.

\section{TABLE I. SYSTEM PARAMETERS}

\begin{tabular}{|c|c|c|c|}
\hline Parameters & Value & Parameters & Value \\
\hline$L_{\mathrm{p}}=L_{\mathrm{s}}=L_{\mathrm{X}}=L_{\mathrm{Y}}=L$ & $12 \mu \mathrm{H}$ & $U_{\mathrm{in}, \mathrm{s}}$ & $1 \mathrm{~V}$ \\
\hline$M$ & $2.5 \mu \mathrm{H}$ & $f$ & $90 \mathrm{kHz}$ \\
\hline$U_{\text {in }}$ & $36.5 \mathrm{~V}$ & $C_{\mathrm{p}}=C_{\mathrm{s}}=\mathrm{C}$ & $0.26 \mu \mathrm{F}$ \\
\hline
\end{tabular}

After the accesses of the legal load to the system, the $\mathrm{S}_{1}$ was turned on under the control of MCU2. In this way, the 
system formed a LCL-LCL topology allowing the CC output and the MCU1 adjusted the input voltage of the system to 36.5 $\mathrm{V}$, so that the system can normally charge the lithium battery. Figure III shows the changes of the charging current and voltage of the battery when the system charges the legal load.

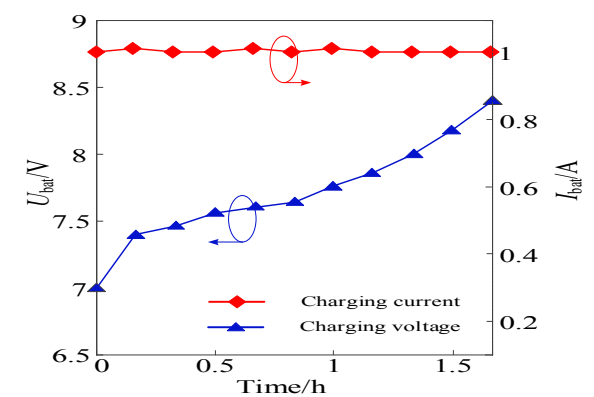

FIGURE III. CHANGES OF THE CHARGING VOLTAGE AND CURRENT OF THE BATTERY WITH TIME

It can be seen that in the $\mathrm{CC}$ mode, the charging current of the battery maintained at $1 \mathrm{~A}$ while the voltage of the battery gradually enlarged with time, till reaching $U_{\text {bat, } s,}$, that is, $8.4 \mathrm{~V}$. According to the above analysis relating the charging strategy of the system, all of the switches in the system were turned off before the access of the battery, the voltage of the power source was the standby voltage, and the voltage of the compensating capacitor $U_{\mathrm{Cp}}$ was equal to that of the power source. After the access of the legal load to the system, the switch $S_{1}$ was turned on, and $U_{\mathrm{Cp}}$ should meet Formula (13). If the battery connected to the system was an illegal load, the switch in the secondary side was still turned off and $U_{\mathrm{Cp}}$ still equaled the standby voltage of the power source. To verify whether Formula (13) is able to identify illegal loads in the system, four groups of lithium batteries of different voltage classes were used in the experiment. The switch $\mathrm{S}_{1}$ was turned on to measure the voltage $U_{\mathrm{Cp}}$ in the two sides of the compensating capacitor $\mathrm{C}_{\mathrm{p}}$ after the access of different batteries to the system with same parameters.

The nominal voltages of the batteries connected to the system were $7.4 \mathrm{~V}, 3.7 \mathrm{~V}, 11.1 \mathrm{~V}$, and $14.8 \mathrm{~V}$ in the A, B, C, and $\mathrm{D}$ groups of experiments, respectively. Among them, the battery in the A group was the legal load corresponding to the system. For a lithium battery with a nominal voltage of $7.4 \mathrm{~V}$, its voltage changes in the range of $7 \sim 8.4 \mathrm{~V}$, that is, $U_{\text {bat,u }}=7 \mathrm{~V}$ and $U_{\text {bat, }}=8.4 \mathrm{~V}$. According to Formula (10), when the voltage of the power source of the system was the standby voltage $U_{\mathrm{in}, \mathrm{s}}$, the output current of the system in CC mode was $I_{\text {bat, }}=0.028 \mathrm{~A}$. Then, the authors defined the detection range of legal loads based on Formula (13). That is, when the voltage $U_{\mathrm{Cp}}$ of the compensating capacitor in the primary side of the system was detected to satisfy Formula (13), the primary side judged that the battery was a legal load. According to the data in Table I, the voltages of the compensating capacitor need to meet $1.65<U_{\mathrm{Cp}}<1.87$ when the battery entering the system is a legal load through calculation. Figure IV displays the detected voltages of the compensating capacitor after the access of the four groups of batteries to the system when the $S_{1}$ was switched on.

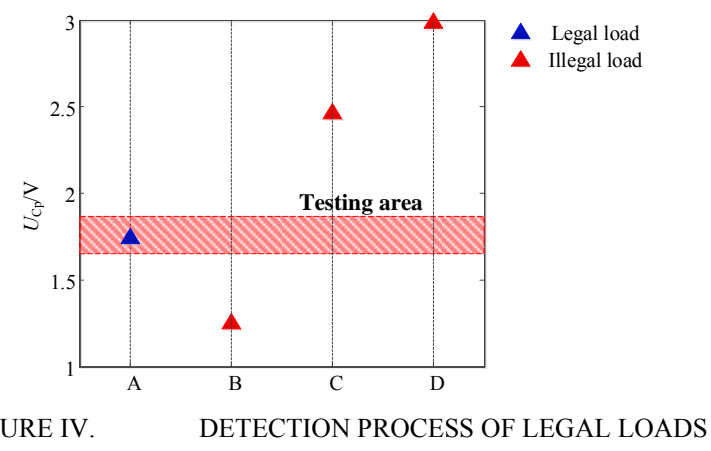

In the A group of experiment, the battery connected to the system had an open-circuit voltage of $7.2 \mathrm{~V}$, and it was detected that $U_{\mathrm{Cp}}=1.69 \mathrm{~V}$, which fell into the detection range of legal loads. Therefore, the battery was judged as a legal load. In the $\mathrm{B}$ group of experiment, the open-circuit voltage of the battery was $4 \mathrm{~V}$ and $U_{\mathrm{Cp}}=1.25 \mathrm{~V}$, which did not satisfy Formula (13), so the system judged that the battery was an illegal load. Similarly, in the C and D groups of experiment, the voltages of the compensating capacitor were measured to be $2.46 \mathrm{~V}$ and $2.98 \mathrm{~V}$, also not meeting Formula (13). Therefore, the system judged that the two batteries were also illegal loads. The comparison between the judgment results of the system and the real conditions of the batteries shows that the designed charging strategy can accurately identify the properties of batteries in the system and thereby improves the safety of the system.

\section{CONCLUSIONS}

The research put forwards the LCL-LCL topology that can realize the $\mathrm{CC}$ output by investigating the output characteristics of the WPT systems with different compensation topological structures. Then, the above topology is applied to the wireless charging of lithium batteries. Based on the above analysis, the authors design the wireless charging system allowing CC charging and propose the charging strategy of wireless charging systems combining the charging characteristics of lithium batteries. The charging strategy not only can guarantee the CC charging of lithium batteries, but also can identify illegal loads in the system and make corresponding responses, thus improving the safety of the system. Finally, the experimental prototype of the system was built to charge the lithium battery with a nominal voltage of $7.4 \mathrm{~V}$ wirelessly. Meanwhile, it is verified that the system can accurately identify illegal loads when they enter the system.

\section{ACKNOWLEDGEMENT}

This research is supported by China Southern Power Grid Corporation (Research and Application of Key Technologies of Intelligent Cloud Service in Hainan Power Grid).

\section{REFERENCES}

[1] X. Huang, W. Wang, L. Tan, "Fast non-intrusive load identification algorithm for resident load based on template filtering," in Automation of Electric Power Systems, vol. 41, no. 2, pp. 2-14, 2017.

[2] Y. Zhai, Y. Sun, X. Dai, et al, "Modeling and analysis of magnetic resonance wireless power transmission systems," in Proceedings of the CSEE, vol. 32, no. 12, pp. 155-160, 2012. 
[3] Q. Yang, P. Zhang, L. Zhu, et al, "Key fundamental problems and technical bottlenecks of the wireless power transmission technology," in Transactions of China Electrotechnical Society, vol. 30, no. 5, pp. 1-8, 2015.

[4] A. Kurs, A. Karalis, Moffatt R, et al, "Wireless power transfer via strongly coupled magnetic resonances," in Science, vol. 317, no.5834, pp. 83-86, 2007.

[5] A. Khaligh, Z. Li, "Battery, Ultracapacitor, Fuel Cell, and Hybrid Energy Storage Systems for Electric, Hybrid Electric, Fuel Cell, and Plug-In Hybrid Electric Vehicles: State of the Art," in IEEE Transactions on Vehicular Technology, vol. 59, no. 6, pp. 2806-2814, 2010.

[6] J. T. Boys, G. A. Covic, Y. Xu, "DC analysis technique for inductive power transfer pick-ups," in IEEE Power Electronics Letters, vol. 99, no. 2, pp. 51-53, 2003.

[7] H. H. Wu, A. Gilchrist, K. D. Sealy ,et al, "A High Efficiency $5 \mathrm{~kW}$ Inductive Charger for EVs Using Dual Side Control," in IEEE Transactions on Industrial Informatics, vol. 8, no. 3, pp. 585-595, 2012.

[8] Z. H. Wang, Y. P. Li, Y. Sun, et al. "Load Detection Model of VoltageFed Inductive Power Transfer System," in IEEE Transactions on Power Electronics, vol. 28, no.11, pp. 5233-5243. 